\title{
Perspectives of research ethics committee members on human challenge studies in the development of vaccines against COVID-19: a mixed methods study
}

\author{
Zihan Pan ${ }^{1 \#}$, Hongling Chu ${ }^{2 \#}$, Yu Zhang ${ }^{3 \#}$, Xue Hong ${ }^{3}$, Lijun Liang ${ }^{3}$, Wenzhi Zhao ${ }^{3}$, Yahong Chen ${ }^{1,3}$, \\ Chunli Song ${ }^{3,4}$
}

${ }^{1}$ Department of Respiratory and Critical Care Medicine, Peking University Third Hospital, Beijing, China; ${ }^{2}$ Research Center of Clinical Epidemiology, Peking University Third Hospital, Beijing, China; ${ }^{3}$ Ethics Committee for Medical Scientific Research, Peking University Third Hospital, Beijing, China; ${ }^{4}$ Department of Orthopedics, Peking University Third Hospital, Beijing, China

Contributions: (I) Conception and design: Y Chen, C Song; (II) Administrative support: Y Chen, C Song; (III) Provision of study materials or patients: Y Chen, C Song; (IV) Collection and assembly of data: Z Pan, H Chu, Y Zhang, X Hong, L Liang, W Zhao; (V) Data analysis and interpretation: Z Pan, H Chu, Y Zhang, X Hong, L Liang, W Zhao; (VI) Manuscript writing: All authors; (VII) Final approval of manuscript: All authors.

\#These authors contributed equally to this work.

Correspondence to: Yahong Chen. Department of Respiratory and Critical Care Medicine, Peking University Third Hospital, Beijing, China.

Email: chenyahong@vip.sina.com; Chunli Song. Department of Orthopedics, Peking University Third Hospital, Beijing, China. Email: schl@bjmu.edu.cn.

Background: Vaccines are considered the most effective protection against the coronavirus disease 2019 (COVID-19). Human Challenge Studies can help to shorten the development process of vaccines. The reviewers' opinions from research ethics committees (REC) play an essential gate-keeping role in determining whether a clinical trial can be conducted or not.

Methods: A convergent mixed-methods study was conducted in a leading general hospital in China. A total of 58 REC members from the institution were invited to participate in an online questionnaire survey. According to the result of the quantitative survey, 15 of these REC members were purposefully selected to participate in qualitative interviews further. Quantitative data were analyzed using descriptive statistical techniques, and thematic analysis was used to analyze the qualitative data. Findings from the quantitative and qualitative analyses were synthesized to deeply illustrate the attitudes, views, and suggestions of REC members on human challenge studies to develop COVID-19 vaccination.

Results: The response rate of the online questionnaire was $62 \%$ (36/58), and 15 of the respondents were interviewed. All participants deemed that the human challenge study should provide compensation to its participants and that sufficiently informed consent is necessary. The human challenge study was disagreed with by $38.9 \%$ of participants. The key points of concern raised were representativeness and fairness of participant selection, benefit, and risk, vulnerable groups, compensation to participants, informed consent, and general view on human challenge studies.

Conclusions: Human challenge studies helped accelerate the development of vaccines for disease control to a certain extent, but the bottom line of medical ethics should not have been broken. At any time, the rights and interests of research participants should come first.

Keywords: Human challenge study; ethics review; mixed methods

Submitted Dec 30, 2020. Accepted for publication Apr 28, 2021.

doi: 10.21037/apm-20-2622

View this article at: http://dx.doi.org/10.21037/apm-20-2622

^ ORCID: Zihan Pan, 0000-0003-4502-1107; Hongling Chu, 0000-0003-1846-9192. 


\section{Introduction}

At the beginning of 2020, the pandemic of coronavirus disease 2019 (COVID-19) quickly swept the world. On 30 January 2020, the World Health Organization (WHO) announced that the pandemic of COVID-19 was a global public health emergency (1). As of 20 March 2021, there have been 121,959,223 confirmed cases of COVID-19 and 2,694,094 deaths globally. The areas or territories of 216 countries have been affected (2). Much remains unknown about COVID-19; however, the current outbreak situation has revealed that developing vaccines to protect the public against COVID-19 is the most profound and immediate technical challenge humanity has ever faced (3). Currently, many countries around the world are actively undertaking vaccine research on COVID-19 (4). Even though countless people have been cured of the disease, prevention is of greater importance than cure, not just from a health perspective but also from the individual patient.

With the spread of the pandemic, research of vaccines against COVID-19 has become increasingly imminent (5). Conventional clinical research of vaccine development usually needs to progress through laborious largescale phase III clinical trials before entering the stage of clinical application. Different models for truncating this process have been proposed as a strategy for accelerating severe acute respiratory syndrome coronavirus 2 (SARSCoV-2) vaccine development, including traditional vaccine development, pandemic vaccine development model, controlled human infection models (6), and so on. The human challenge study was one of the most controversial of these models. Human challenge studies involved the intentional exposure of study participants to infection with disease-causing pathogens to assess potential vaccine or drug candidates or understand how humans are protected from or acquire the infection (7-11). The concept of giving a healthy person a disease did not align with the public's previous expectations of medicine and has thus triggered a heated discussion. A human challenge study is not without risks, but every week of the SARS-CoV-2 vaccine rollout delay foreshadows many thousands of deaths globally. Importantly, challenge studies are usually conducted based on competent volunteers' informed consent, minimization of study risks, and high baseline risks of infection for participants. They do not violate participants' rights on the alteration of emergency response but heed both individual rights and the global public health emergency. To further assess the potential of human challenge studies to speed vaccine development, researchers have suggested that an expert group might be convened, including those with experience of human challenge studies of other pathogens, regulators, vaccine trialists, ethicists, potential participants, and relevant funding agencies, to plan if and how such studies might be taken forward ethically and expeditiously (12-15). The research ethics committee (REC) is one of the key gatekeepers to determining whether a clinical trial can be conducted or not, and reviewers' standpoints play an essential role. A mixed-methods integrated qualitative and quantitative approach was applied to investigate attitudes, views, and suggestions on human challenge studies from the perspective of a local Chinese REC.

We present the following article in accordance with the SURGE reporting checklist (available at http://dx.doi. org/10.21037/apm-20-2622).

\section{Methods}

\section{Design}

Convergent mixed methods (16) approach integrating a quantitative online survey and qualitative in-depth interviews were used to investigate the attitudes, views, and suggestions of REC members on implementing human challenge studies to develop COVID-19 vaccines. This study was approved by the REC at Peking University Third Hospital [2020-17901]. The study was conducted following the Declaration of Helsinki (as revised in 2013). The summary of the research design is presented in Figure 1.

\section{Participants}

The REC of Peking University Third Hospital, one of the top medical institutions in China, was composed of a group of experts and professionals with diverse academic backgrounds at the time of this study. The committee members majored in 24 clinical specialties, including internal medicine, surgery, pediatrics, gynecology, and obstetrics, as well as law, administration/management, and engineering. All 58 prevailing REC members were invited to this study through e-mails or the social media platform WeChat.

An anonymous online survey was distributed via e-mail or WeChat. At the end of the questionnaire, respondents were asked to supply their contact details if they were willing to take part in an online interview further. A total of 36 of the 58 committee members completed the online 
Quantitative purpose

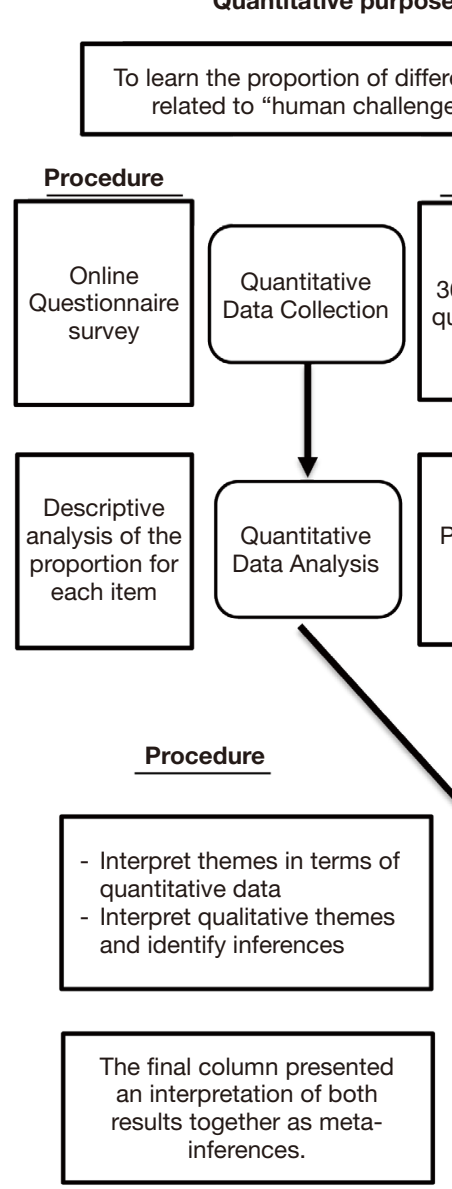

Qualitative purpose

To deeply learn the qualitative views related to "human challenge trial"

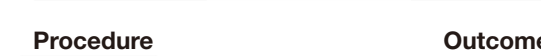

Outcome
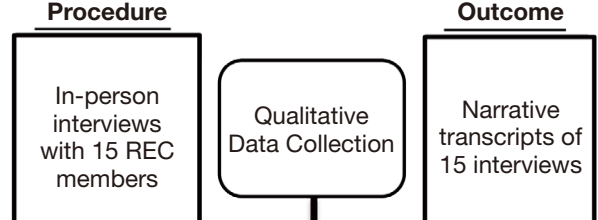

completed

questionnaires

members
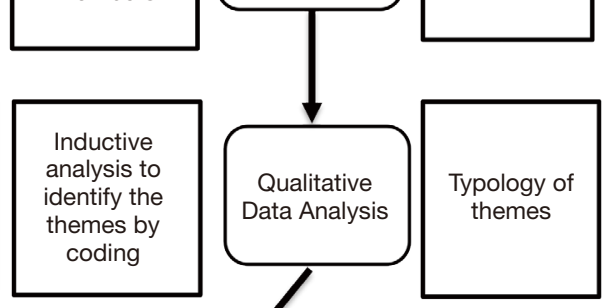

roportion for each item

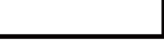

coding

Figure 1 Diagram of convergent mixed methods design for this study.

survey. We purposefully selected 15 of these REC members to participate in qualitative interviews according to their quantitative survey results to reflect different opinions.

\section{Data collection}

This study was conducted from April 2020 to June 2020 .

The data sources are presented in Table 1 .

\section{Quantitative data: online questionnaire survey}

A questionnaire was drawn up by the research team based on a comprehensive literature review of human challenge studies and pilot tested to ensure clarity and understanding. Then, the questionnaire with 22 items, comprising both quantitative and open-ended qualitative questions about the ethics reviewers' attitudes, views, and suggestions on the implementation of human challenge studies, was distributed to all REC members at Peking University Third Hospital through Wenjuanxing Online Survey (17), an online crowdsourcing platform. Participants who voluntarily submitted questionnaires after being informed about the study were considered to have provided consent to participate in the study.

\section{Qualitative data: deptb interviews}

Interview guides were developed according to the domains of the quantitative questionnaire and modified after a pilot interview. The main content of the interview included reviewers' attitudes, views, and suggestions on this controversial issue. In response to the COVID-19 outbreak, interviews were conducted online via the WeChat application with 15 REC members. Interviews 
Table 1 Data sources of quantitative and qualitative phase

\begin{tabular}{lccc}
\hline General type & Who/what (sampling strategy) & How many & Comments \\
\hline $\begin{array}{l}\text { Quantitative data sources: } \\
\text { online questionnaire survey }\end{array}$ & $\begin{array}{c}\text { The whole committee members of the Ethics } \\
\text { Review Board at Peking University Third Hospital }\end{array}$ & 36 & $\begin{array}{c}\text { To learn the proportion of different views } \\
\text { related to "human challenge trial" }\end{array}$ \\
$\begin{array}{l}\text { Qualitative data sources: in- } \\
\text { depth interviews }\end{array}$ & $\begin{array}{c}\text { Committee members were purposeful chosen } \\
\text { based on the result of questionnaire survey }\end{array}$ & 15 & $\begin{array}{c}\text { To deeply learn the qualitative views } \\
\text { related to "human challenge trial" }\end{array}$ \\
\hline
\end{tabular}

were stopped at 15 when themes were saturated; that is, information power was deemed to be enough to explain the research question (18). All interviewees provided oral informed consent to participate in interviews, including permission to audiotape and transcribe the interviews. Potentially identifying information was removed from each transcript.

\section{Statistical analysis}

Quantitative data were analyzed using basic statistical techniques with SPSS version 24 (IBM Corp., Armonk, NY, USA). The number of participants and percentage for each item were uses to describe the distribution for each question in quantitative survey.

\section{Qualitative data analysis}

Inductively thematic analysis was applied to qualitative data using Nvivo 11 (QSR International Pty Ltd., Burlington, MA, USA) (19). The transcripts were coded and analyzed by 3 researchers (HC, ZP, YZ) by: making sense of the transcribed data, developing codes, categorizing the data, and abstracting (20). During this analytic process, 6 key themes were refined as below: representativeness and fairness of subjects selection, benefit and risk, vulnerable groups, compensation to subjects, informed consent, and general view on human challenge studies.

\section{Mixed data analysis}

The mixed data were analyzed based on dimensions using joint display analysis (21). The joint display analysis procedures involved simultaneously both types of data, finding linkages between the 2 types of data, and iteratively developing an optimized table for understanding, interpreting, and presenting the data. This iterative process of juxtaposing related quantitative and qualitative data together, examining the implications of each together, looking for new organizations facilitated the drawing of conclusions in light of both types of findings.

\section{Results}

Of the 58 REC members, 36 (36/58, 62\%) completed the online survey, 13 were male $(36.1 \%)$, and 23 were female $(63.9 \%)$. The duration of years of practice among respondents in the ethics review ranged from 8 months to 25 years. There were 15 participants involved in the qualitative interview, of whom 11 were female $(11 / 15$, $73.3 \%)$, and 4 were male $(4 / 15,26.7 \%)$. The average time of the interview was $33.7 \mathrm{~min}(12-51 \mathrm{~min})$. The demographic information of interviewees is shown in Table 2.

The results from the online survey and interviews were integrated and presented under 6 major themes that emerged from the inductive qualitative analysis, with supporting statistics from the quantitative analysis, which were: (I) representativeness and fairness of participants selection; (II) benefits and risks for participants; (III) vulnerable groups; (IV) compensation to participants; (V) informed consent; (VI) general view on human challenge studies. These dimensions are presented in the joint display (Table 3) and the following paragraphs.

\section{Quantitative findings}

The quantitative survey revealed that $47.3 \%$ of REC members believed the representativeness and fairness of participant selection should be considered; $41.7 \%$ expressed a belief that there is no personal benefit in the human challenge study; $27.8 \%$ believed that vulnerable groups could be considered for the study. All respondents believed that the human challenge study should provide compensation to participants. Sufficiently informed consent is necessary. Among the respondents, $38.9 \%$ disagreed with the human challenge study. Table 4 shows the detailed proportion of opinion 
Table 2 The demographic information of interviewees

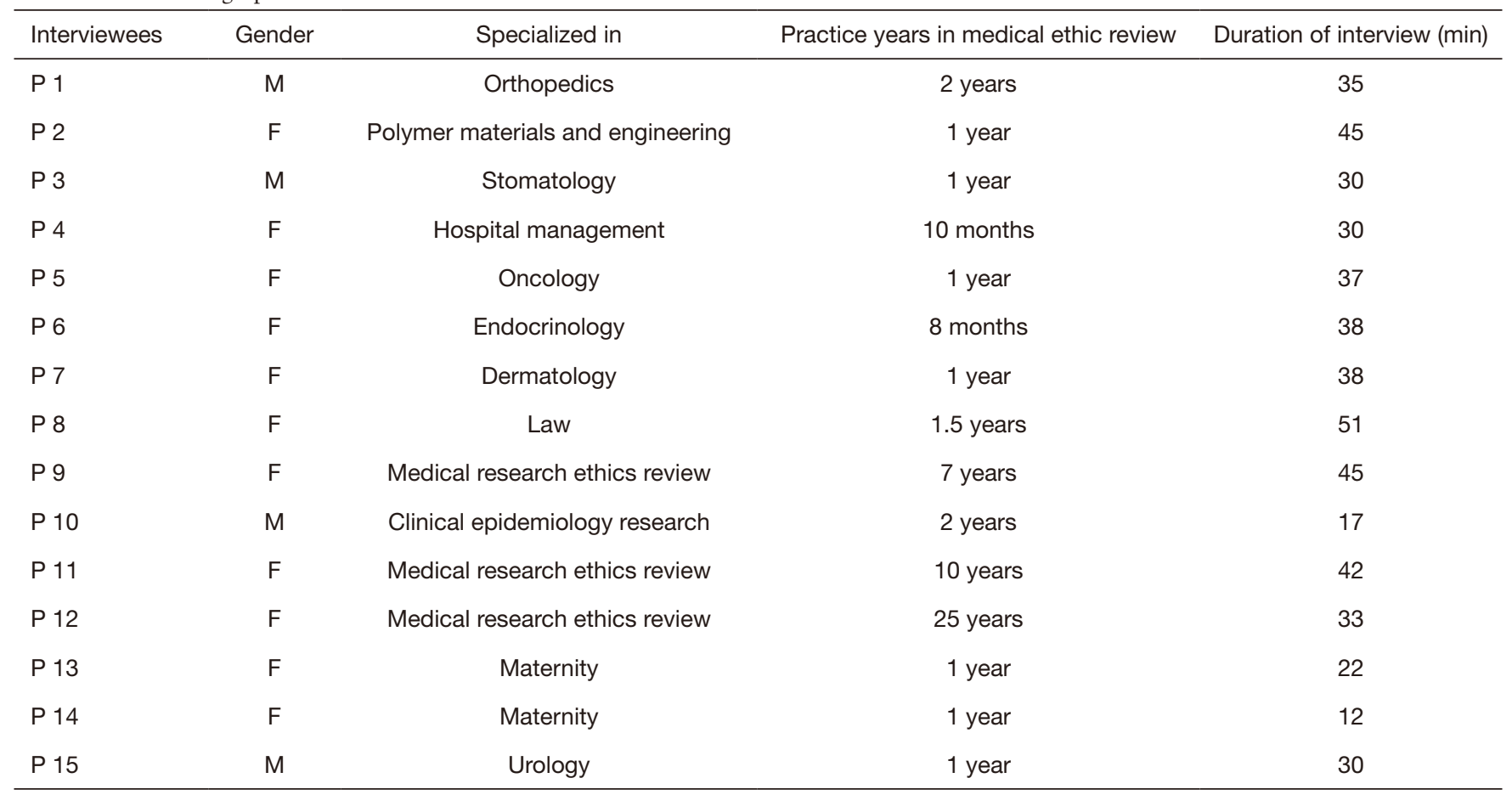

for each dimension.

\section{Integrating the quantitative and qualitative results}

\section{Representativeness and fairness of participant selection}

In the questionnaire survey, $52.8 \%$ of the respondents $(19 / 36)$ thought that it was unreasonable to select healthy people aged $20-45$ years as study participants. From the interviews, however, given that people in this age range were capable of autonomous behavior, and the risk faced by this group of people in trials was lower than that of other people, some interviewees considered that people in this age group were suitable for selection in the human challenge study. Nevertheless, opponents believed that sufficient knowledge of COVID-19 was lacking; although people in this age group were robust, the susceptible population groups in COVID-19 were uncertain, so people in this age group could not represent all people.

Notably, there was one interviewee who believed that the extraordinary nature of the COVID-19 pandemic sufficiently negated the need to place special emphasis on the fairness and representativeness of the study participants: P10 (M, 2 years of practice in the REC) "Now it seems that anyone can be infected with COVID-19, if the vaccine is eventually available to the public, then everyone will be recipients. In such a special period, any subject is representative and fair. So I do not think the special emphasis should be placed on representativeness. Fairness is not that important by the moment."

\section{Benefit and risk}

In the questionnaire survey, 15 (41.7\%) respondents thought that the participants gained no benefits. Interviewees believed that the benefits of vaccinations were twofold, with gains for the individual and for society. If the COVID-19 vaccination were successfully developed and had a protective effect, the individual would benefit after vaccination, but society would benefit in a greater capacity, or the social benefits brought by the vaccination far outweighed the benefits of individuals.

Some interviewees even believed that participants gained no personal benefits in these trials, only social benefits, while on the other hand, they would bear huge risks. The vaccination itself could also be seen to bring risk to participants, for example, in the form of side effects.

It was also revealed that interviewees thought that there was a worldwide lack of knowledge and treatment for COVID-19, so there was also a risk of uncertainty among 
Table 3 Joint display of quantitative and qualitative findings by 6 dimensions

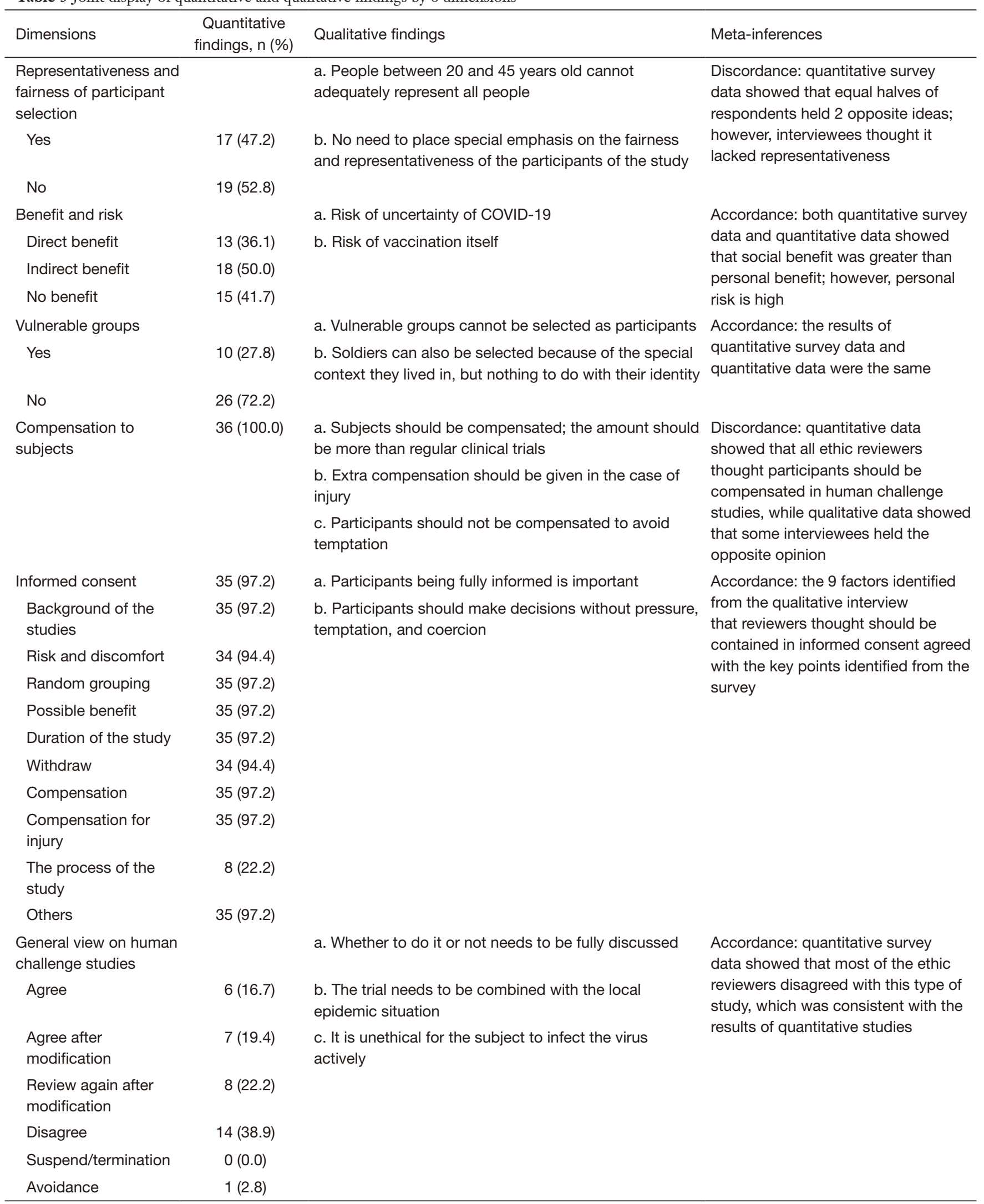


Table 4 Result of the quantitative survey from 6 dimensions

\begin{tabular}{|c|c|}
\hline Dimensions & Proportion, n (\%) \\
\hline \multicolumn{2}{|c|}{ Representativeness and fairness of subject selection } \\
\hline Yes & $17(47.2)$ \\
\hline No & $19(52.8)$ \\
\hline \multicolumn{2}{|l|}{ Benefit and risk } \\
\hline Direct benefit & $13(36.1)$ \\
\hline Indirect benefit & $18(50.0)$ \\
\hline No benefit & $15(41.7)$ \\
\hline \multicolumn{2}{|l|}{ Vulnerable groups } \\
\hline Yes & $10(27.8)$ \\
\hline No & $26(72.2)$ \\
\hline Compensation to subjects & $36(100.0)$ \\
\hline Informed consent & $35(97.2)$ \\
\hline Background of the studies & $35(97.2)$ \\
\hline Risk and discomfort & $34(94.4)$ \\
\hline Random grouping & $35(97.2)$ \\
\hline Possible benefit & $35(97.2)$ \\
\hline Duration of the study & $35(97.2)$ \\
\hline Withdraw & $34(94.4)$ \\
\hline Compensation & $35(97.2)$ \\
\hline Compensation for injury & $35(97.2)$ \\
\hline The process of the study & $8(22.2)$ \\
\hline Others & $35(97.2)$ \\
\hline \multicolumn{2}{|c|}{ General view on human challenge studies } \\
\hline Agree & $6(16.7)$ \\
\hline Agree after modification & $7(19.4)$ \\
\hline Review again after modification & $8(22.2)$ \\
\hline Disagree & $14(38.9)$ \\
\hline Suspend/termination & $0(0.0)$ \\
\hline Avoidance & $1(2.8)$ \\
\hline
\end{tabular}

the global population. Even so, interviewees thought that personal interests must not be compromised for the benefit of society, such an idea was unethical, and we should keep in mind the first medical maxim of causing "no harm." Minimizing the risk to participants in these trials was crucial issue.

Participant 10 (M, 2 years of practice in the REC) expressed the following belief: "I personally think that in fact, the subject has no personal benefit, it may be more of a benefit to a group outside the scope of the beneficiary, a benefit to the whole society, and the subject may bave more personal benefits. More is in this level of social recognition and personal honor, of course, be will also have a better self-identity, which may be his potential benefit."

Another respondent (P11, F, 10 years of practice in the REC) expressed themselves like this: "At this time, be bears a double risk, not only the risk of exposure, but also the impact of the vaccination itself, or some subsequent side effects."

\section{Vulnerable groups}

A total of 10 respondents $(27.8 \%)$ in the questionnaire survey thought that vulnerable groups (for example, prisoners), or those who were susceptible to the virus could be selected in this case; while $26(72.2 \%)$ respondents considered that such populations could not be selected in this case. In the interview, in terms of participant recruitment, interviewees thought they should not recruit vulnerable groups, not only because the living environment and psychological state of the vulnerable groups could not adequately match healthy people, but also harmed the rights and interests of such participants. Also, recruiting the vulnerable was unethical, discriminatory, and would infringe on their human rights. Some interviewees believe that if a disadvantaged population were to be involved in such a human challenge study, it could only be appropriate if their living environment was suitable for the study, rather than as a result of their identification as a vulnerable population.

Insight from another REC member (P6, F, 8 months of practice in the REC) was expressed as follows: "I think the human challenge study absolutely cannot involve vulnerable groups. First of all, the buman challenge study is a very controversial type of clinical trial to intentionally create infection to harm subjects, which challenges ethics and morals even when normal and healthy people are involved. Even if the subject is young, healthy, and with the lowest morbidity or mortality, they can still have a moral defense to a certain extent; but ethical fairness and respect would be challenged if involving vulnerable people. It must be resolutely avoided in the challenge experiment."

\section{Compensation to participants}

A total of $26(72.2 \%)$ respondents supported the provision 
of compensation and that it should be given according to the standard of no less than phase I clinical study. In comparison, $10(27.8 \%)$ people considered that compensation should be given following the standards of general clinical studies to avoid overly incentivizing participants. Overall, the online questionnaire demonstrated that all members upheld that the participants be compensated. Some interviewees believed that the participants should be compensated; free treatment should be provided to them, but some mental impact compensation should be provided, as emotional responses such as anxiety might occur after being exposed to the infection. In this case, compensation could be used for counseling and therapy or make things a little bit easier to assimilate mentally.

Regarding the amount of participant compensation, it should at least match that of phase 1 clinical trial, perhaps even more, as it is a human challenge trial, the compensation should also be "challenging." However, some interviewees believed that no extra compensation should be awarded in addition to the basic subsidy. The extra compensation may invoke a certain temptation to participate in such a study, which might compromise ethical fairness. Therefore, the decision of participation should be made based on willingness after full informed consent and without compensation.

A respondent (P11, F 10 years of practice in the REC) expressed her thoughts on compensation as follows: "From my point of view, I do not recommend providing this kind of compensation, like in our daily research. Because this kind of research is true of a very special design, even if it is such a bealthy adult, various economic factors may affect his willingness to participate in the research. Therefore, if he really is willing to participate in such a study, he could make a decision based on providing full informed consent and without any financial inducement."

\section{Informed consent}

Interviewees believed that it was essential to ensure that participants were fully informed; consequently, they could make decisions based on a clear understanding of the study and associated risks. The interviewees emphasized that in such a special study, it was not enough for participants to provide informed consent to subjects and their relatives.

Thoughts were expressed as such (P7, F, practiced 1 year in REC): "Subjects should be given sufficient information of the study including the aim, process, what will be required of participants, and any risks that they will face, for their notification and consideration. I think the consent of immediate family members is also very important."

\section{General view on human challenge studies}

In the questionnaire survey, some respondents (38.9\% (14/36) disagreed with challenge studies in the context of COVID-19. Further qualitative interviews gleaned that some interviewees thought that the decision needs to be combined with the local epidemic situation. Meanwhile, some other interviewees thought that as there were still many unknowns about the virus, a human challenge study was not feasible, and it was unethical to infect people with a disease purposively.

Such sentiments were captured as such: $(\mathrm{P} 2, \mathrm{~F})$ : "The study of vaccination via human challenge events is still a bit doubtful, especially for bealtby people. My biggest doubt is to do controlled experiments on healthy people; that is to say, they were not yet vaccinated but exposed to the virus. I am a little against this."; (P15 M): "As a REC member, I would disagree with this study because, for the subject, this poses such a risk of excessive physical damage."; (P11 F): "If this research is going to be conducted in China, I do not agree. If we do one, that is, where the epidemic is serious, I may agree."

Quantitative and qualitative results are shown below by joint display in Table 2 .

\section{Discussion}

In the current urgent pandemic situation, and under the boost of public opinion, it became less complicated to issue passes for human challenge tests; whereas, we would do better to evaluate the challenge test with objectiveness and fairness. This mixed-methods study presented the views and opinions of REC members from one of China's top research medical institutions regarding human challenge studies.

The Peking University Third Hospital, founded in 1958, is a first-class general hospital integrating medical practice, teaching, scientific research, prevention, rehabilitation, and health care, with more than 2,000 beds and 37 medical departments. The institution has achieved remarkable results in the field of medical research (22). Peking University Third Hospital has a well-established and mature medical ethics review system behind these outstanding medical research achievements. The perspectives and opinions in this study can represent the overall attitude of the hospital's REC towards human challenge studies to a 
certain degree; however, other ethical reviewers may still offer different perspectives. The outbreak news stated that the world's first COVID-19 vaccine was registered on 11 August 2020 in Russia, which came as a shock to the world. Undeniably, it was a breakthrough in the global response to the COVID-19 pandemic; however, skipping the phase III trial also stirred deep concerns regarding the vaccine's safety, effectiveness, and developmental procedure. We should note other COVID-19 vaccines were also entered into Phase III trials before receiving emergency use authorization (EUA). Increasing studies relevant to the COVID-19 vaccine have moved forward, but the ethical issues should not be diluted, and serious discussions need to be raised even though the COVID-19 pandemic has represented a situation of emergency.

The consensus of the interviewees in this study was that uniform principles of ethics in medical research should be observed invariably regardless of circumstances which were consistent with the views of some academics who have asserted that challenge studies need to be undertaken within the general regulatory framework of medical research (23). In terms of scientificity, the interviewees found it scientifically neglectful to skip phase III clinical trials in vaccine research and development, and they believed that the science of COVID-19 vaccines was being compromised to favor speed over public welfare. Regarding ethics, concerns about challenge studies mainly derived from the fact that they involved infecting healthy people with COVID-19 when there was a shortage of therapeutic methods for COVID-19. Therefore, the core ethical issue concerning challenge studies was in the risk of harm to the participants.

Most of the results of the qualitative and quantitative sections complemented each other except for in the compensation of participants. In the questionnaire survey, all respondents answered that participants should be compensated; however, in the interview, some opinions were voiced that no compensation should be given to participants in case of incentivization. The latter believed that participating in these studies without any compensation can eliminate participant temptation and that there was a possibility that participants might be unduly enticed to participate by offers of (excessive) payment.

Human challenge studies have been an important strand of much current research, particularly in the development of vaccines, such as those against malaria, cowpox, and yellow fever. Medical research has a long history of intentionally infecting healthy people to study diseases, such as challenge studies with influenza A virus, cholera bacilli, and pneumococcus (23). Many factors need to be considered when considering whether the public would accept or conduct human challenge studies, such as general ethical principles, local epidemic situation, and cultural characteristics. Human challenge studies can facilitate disease control to a certain extent (23), but the bottom line of medical ethics should not be crossed. At any time, the rights and interests of the participants should come first. Challenge studies could be conducted ethically without lowering scientific and medical ethical standardsthey merit serious consideration as the human toll of the COVID-19 pandemic continues to grow, which was similar to the results of our study (15).

China was hit with COVID-19 at the end of 2019, and while actively fighting against the pandemic, researchers from China were also accelerating the pace of clinical research on COVID-19. As of 13 August 2020, there are 167 vaccine candidates for COVID-19, 138 candidate vaccines were in preclinical evaluation, 29 candidate vaccines were in clinical evaluation, among which 6 were in phase III clinical trials with an advanced speed, and 2 of those 6 were from China (4). Although China has faced a heavy workload in scientific research to combat COVID-19, the country's efforts have not slackened in terms of ethical review. In terms of clinical research related to COVID-19, China has always adhered to the following principles: (I) strictly abide by the standard of censorship; (II) guarantee the rights and interests of the participants; (III) guarantee the independence of the ethics committee and ensure that it conducts the review independently; (IV) achieve full ethical coverage and supervision of key projects that can be tackled in an emergency; (V) improve the efficiency of review $(24,25)$. The $\mathrm{WHO}$ has set forth 8 essential criteria for conducting SARS-CoV-2 challenge studies (26). In this special time, when researching COVID-19 in China, Chinese academics who devote themselves to COVID19-relevant medical research do so in adherence with both local and international medical ethics review requirements $(26,27)$.

\section{Strength and limitation of the study}

This study integrated a quantitative survey and qualitative interview to investigate the attitudes and perspectives on human challenge studies of experts in the medical ethics review board. The results of this study illustrated the position of experts working in a medical ethics review board 
in China, especially in an emergency. This study had some limitations. Firstly, our study was limited to the attitudes and views of the medical ethics committee members in a single top-level institution in China. The views of other professional stakeholders, like non-university hospitals, biotechnology companies, the general public, and people in the community in China may be more diverse. Secondly, as the study was conducted during a period when the COVID-19 pandemic was controlled relatively well in China, participants' concerns may have been affected by the pandemic situation. Thirdly, even though the participants in this study had various backgrounds, all were from the same institution. This study involved an exploratory investigation into the perspectives of REC members on human challenge studies in the development of vaccines against COVID-19 with a small sample size; therefore, the generalizability of this study is limited. If required, a cross-sectional survey applied to a large sample of high quality could achieve good generalizability.

\section{Acknowledgments}

We thank our colleagues at the Peking University Third Hospital who provided detailed comments on developing the questionnaires and topic guide. We thank all the respondents of the questionnaire and interviewees. Special thanks to Rachel Adams (University of Birmingham) and Alicia O'Cathain (University of Sheffield, UK), who provided constructive suggestions.

Funding: The study was funded by Capital Health Development Research Project (2020-2Z-40917).

\section{Footnote}

Reporting Checklist: The authors have completed the SURGE reporting checklist. Available at http://dx.doi. org/10.21037/apm-20-2622

Data Sharing Statement: Available at http://dx.doi. org/10.21037/apm-20-2622

Peer Review File: Available at http://dx.doi.org/10.21037/ apm-20-2622

Conflicts of Interest: All authors have completed the ICMJE uniform disclosure form (available at http://dx.doi. org/10.21037/apm-20-2622). The authors have no conflicts of interest to declare.
Ethical Statement: The authors are accountable for all aspects of the work in ensuring that questions related to the accuracy or integrity of any part of the work are appropriately investigated and resolved. The study was conducted following the Declaration of Helsinki (as revised in 2013) and was approved by the Ethics Committee of the Research Ethics Committee at Peking University Third Hospital [2020-17901]. Participants who voluntarily submitted questionnaires online after being informed about the study were considered to have agreed to participate in the quantitative survey. In qualitative interviews, oral informed consent was taken through the recording at the beginning of the interview.

Open Access Statement: This is an Open Access article distributed in accordance with the Creative Commons Attribution-NonCommercial-NoDerivs 4.0 International License (CC BY-NC-ND 4.0), which permits the noncommercial replication and distribution of the article with the strict proviso that no changes or edits are made and the original work is properly cited (including links to both the formal publication through the relevant DOI and the license). See: https://creativecommons.org/licenses/by-nc-nd/4.0/.

\section{References}

1. Coronavirus disease (COVID-19) pandemic. Available online: https://www.who.int/emergencies/diseases/novelcoronavirus-2019

2. COVID-19 vaccines. Available online: https://www.who. int/emergencies/diseases/novel-coronavirus-2019/covid19-vaccines

3. Jamrozik E, Selgelid MJ. COVID-19 human challenge studies: ethical issues. Lancet Infect Dis 2020;20:e198-e203.

4. Draft landscape and tracker of COVID-19 candidate vaccines. Available online: https://www.who.int/ publications/m/item/draft-landscape-of-covid-19candidate-vaccines

5. Zhu FC, Li YH, Guan XH, et al. Safety, tolerability, and immunogenicity of a recombinant adenovirus type5 vectored COVID-19 vaccine: a dose-escalation, openlabel, non-randomised, first-in-human trial. Lancet 2020;395:1845-54.

6. Deming ME, Michael NL, Robb M, et al. Accelerating Development of SARS-CoV-2 Vaccines-The Role for Controlled Human Infection Models. N Engl J Med 2020;383:e63. 
7. Njue M, Njuguna P, Kapulu MC, et al. Ethical considerations in Controlled Human Malaria Infection studies in low resource settings: Experiences and perceptions of study participants in a malaria Challenge study in Kenya. Wellcome Open Res 2018;3:39.

8. Expert Committee on Biological Standardization. Human Challenge Trials for Vaccine Development: regulatory considerations. World Health Organization 2016. Available online: https://www.who.int/biologicals/expert_ committee/Human_challenge_Trials_IK_final.pdf

9. Gordon SB, Rylance J, Luck A, et al. A framework for Controlled Human Infection Model (CHIM) studies in Malawi: Report of a Wellcome Trust workshop on CHIM in Low Income Countries held in Blantyre, Malawi. Wellcome Open Res 2017;2:70.

10. World Health Organization. Standards and Operational Guidance for Ethics Review of Health-Related Research with Human Participants. World Health Organization 2011. Available online: https://www.who.int/ethics/ publications/9789241502948/en/

11. Darton TC, Blohmke CJ, Moorthy VS, et al. Design, recruitment, and microbiological considerations in human challenge studies. Lancet Infect Dis 2015;15:840-51.

12. Eyal N, Lipsitch M, Smith PG. Human challenge studies to accelerate coronavirus vaccine licensure. J Infect Dis 2020;221:1752-6.

13. Shah SK, Miller FG, Darton TC, et al. Ethics of controlled human infection to address COVID-19. Science 2020;368:832-4.

14. Plotkin SA, Caplan A. Extraordinary diseases require extraordinary solutions. Vaccine 2020;38:3987-8.

15. Schaefer GO, Tam CC, Savulescu J, et al. COVID-19 vaccine development: Time to consider SARS-CoV-2 challenge studies. Vaccine 2020;38:5085-8.

16. Fetters MD, Curry LA, Creswell JW. Achieving

Cite this article as: Pan Z, Chu $\mathrm{H}$, Zhang $\mathrm{Y}$, Hong $\mathrm{X}$, Liang L, Zhao W, Chen Y, Song C. Perspectives of research ethics committee members on human challenge studies in the development of vaccines against COVID-19: a mixed methods study. Ann Palliat Med 2021;10(6):6259-6269. doi: 10.21037/ apm-20-2622 integration in mixed methods designs-principles and practices. Health Serv Res 2013;48:2134-56.

17. Wenjuanxing (software). Available online: https://www.wjx. $\mathrm{cn} /$

18. Guest G, Bunce A, Johnson L. How many interviews are enough? An experiment with data saturation and variability. Field methods 2006;18:59-82.

19. Braun V, Clarke V. Using thematic analysis in psychology. Qual Res Psychol 2006;3:77-101.

20. Elo S, Kyngas H. The qualitative content analysis process. J Adv Nurs 2008;62:107-15.

21. Fetters MD. The mixed methods research workbook: Activities for designing, implementing, and publishing projects. Thousand Oaks: SAGE Publications; 2020.

22. Peking University Third Hospital. Available online: https://www.puh3.net.cn/index.shtml

23. Hope T, McMillan J. Challenge studies of human volunteers: ethical issues. J Med Ethics 2004;30:110-6.

24. Measures for the Ethics Review of Biomedical Research involving Human beings. Available online: http://www.gov. cn/gongbao/content/2017/content_5227817.html

25. Regulations for the Quality Management of Clinical Trials of Drugs (No. 57, 2020). Available online: https://www. nmpa.gov.cn/yaopin/ypggtg/20200426162401243.html

26. World Health Organization Advisory Group on Human Challenge Studies. Feasibility, potential value and limitations of establishing a closely monitored challenge model of experimental COVID-19 infection and illness in healthy young adult volunteers. World Health Organization 2020. Available online: https://www.who.int/ publications/m/item/

27. Key criteria for the ethical acceptability of COVID-19 human challenge studies. Available online: https://apps. who.int/iris/bitstream/handle/10665/331976/WHO2019-nCoV-Ethics_criteria-2020.1-eng.pdf?ua=1 